\title{
New Time-Frequency Distribution
}

Soo-Chang Pei and Er-Jung Tsai

Institute of Electrical Engineering

National Taiwan University

Taipei, Taiwan, Republic of China

Abstract--The cross terms are an inherent consequence of the second order nature of Cohen's class TFDs (Time Frequency Distribution) [1], [2]. They are manifest in a TFD of multi-component signals as spurious artifacts arising from interactions between the various signal components, and they can often appear at times and/or frequencies inconsistent with the underlying physical nature of the signal as a result to misinterpretation [3], [4], [5]. There are so many time frequency distributions to avoid cross term effect, the best are ChoiWilliams ED (Exponential Distribution) [6] and Levin's IPS (Instantaneous Power Spectrum) [7]. In this paper, we combine the cross term reducing philosophy of $E D$ and IPS, to obtain a new TFD which most effectIy reduce the cross term. Surprising, the new TFD also satisfies most desired properties of TFD.

\section{INTRODUCTION}

The cross terms are an inherent consequence of the second order nature of Coher's class TFD's. They are manifest in a TFD of multi-component signals as spurious artifacts arising from interactions between the various signal components, and they car often appear at times and/or frequencies inconsistent with the underlying physical nature of the signal as a result to misinterpretation.

Generally speaking, there are two alternative methods to control cross terms interference:

(1) Interference attenuation: For example, ChoiWilliams exponential distribution [6] effectively attenuates cross intenference including satisfying many desirable properties of time-frequency distribution.

(2) Interference concentration: For example, Levin's IPS [7] produces cross terms only at signal frequercies, and only during time intervals that the signal is nonzero.

Ir this paper, we will promote a new time-frequercy distribution, which not orly can meet interference attenuation constrairt but also can approximately satisfy interference concentration constraint including satisfying many useful time-frequency distribution properties [2], $[8]$ and $[9]$. DESIGN PHILOSOPHY

First, we recall the definition of Cohen's class [1] for signal $s(t)$ :

$$
\begin{aligned}
& P(t, \omega)=\frac{T}{2 \pi} \iint S e^{-j \theta t-j \omega \tau+j \theta \mu} \phi(\theta, \tau) s\left(\mu+\frac{1}{2} \tau\right) s^{*}\left(\mu-\frac{1}{2} \tau\right) d \mu d \tau d \theta(1) \\
& =\frac{1}{2 \pi} \int S \Psi(t-\mu, \tau) S\left(\mu+\frac{1}{2} \tau\right) s^{*}\left(\mu-\frac{1}{2} \tau\right) e^{-j \omega \tau} d \mu d \tau \\
& =\frac{1}{4 \pi^{2}} \iint_{\varphi}(\theta, \omega-\eta) S^{*}\left(n+\frac{1}{2} \theta\right) S\left(n-\frac{1}{2} \theta\right) e^{-j \theta t} d n d \theta \\
& =\int \delta \Phi(\theta, \tau) A_{S}(\theta, \tau) e^{-j \theta t-j \omega \tau} d \theta d t \text {. } \\
& \text { where } \\
& s(\omega)=\int s(t) e^{-j \omega t} d t \\
& \Psi(\mu, \tau)=\int \Phi(\theta, \tau) e^{-j \theta \mu} \mathrm{d} \theta \\
& \varphi(\theta, n)=\delta \Phi(\theta, \tau) e^{-j \eta \tau} d \tau
\end{aligned}
$$

$0-7803-1254-6 / 93 \$ 03.00 \oplus 1993$ IEEE

$$
A_{S}(\theta, \tau)=\frac{1}{2 \pi} \delta_{S}\left(\mu+\frac{1}{2} \tau\right) S^{*}\left(\mu-\frac{1}{2} \tau\right) e^{j \theta_{\mu}} d \mu
$$

$A_{s}(\theta, \tau)$ is the ambiguity function of $s(t)$, and $\phi(\theta, \tau)$ is an arbitrary function called the kernel function by Claasen [5]. Kernel function of Coher's class is the key to properties of TFD. The properties of TFD can be corresponding to constraints on kernel function $[2],[9]$ and $[10]$.

P. Flandrin [8] pointed out that the desired components of the ambiguity function fall along the $\theta, \tau$ axes in $(\theta, \tau)$ plane. So, the interference atteruation constraint required that

$\Phi(\theta, \tau)$ is low-pass in $(\theta, \tau)$

P.J. Loughlin [9] pointed out that the constraints or $\Phi(\theta, \tau)$ for preventing cross term appearing at nonsignal frequencies and appearing in time intervals where signal is zero in TFD are

$$
\begin{aligned}
& \varphi(\theta, \eta)=0, \text { for }|\eta| \pm \frac{1}{2}|\theta|, \\
& \Psi(\mu, \tau)=0, \text { for }|\mu| \neq \frac{1}{2}|\tau|
\end{aligned}
$$

Eq.(10),(11) are interference concentration constraint. Interesting, the kernel function of $\mathrm{ED}[6]$,

$$
\Phi \operatorname{ED}(\theta, \tau)=e^{-\frac{\theta^{2} \tau^{2}}{\sigma}}
$$

strongly satisfies the interference attenuation constraint eq.(9) and the kernel function of IPS [7]

$$
\Phi_{\operatorname{IPS}}(\theta, \tau)=\cos \left(\frac{1}{2} \theta \tau\right)
$$

meets the interference concentration constraint eq. (10), (11). Now, we think how about a kerrel function equal to product of ED's and IPS's kemel function. Will the new TFD have strongly anti-interference effect? Next, we will prove that the new TFD not only has least interference effect but also meets more useful properties of TFD.

\section{THE NEW TIME-FREQUENCY DISTRIBUTION}

We define the kernel function of the new TFD to be product of ED [6] and IPS [7].

$$
\Phi_{\mathrm{NEW}}(\theta, \tau)=e^{-\frac{\theta 2 \tau 2}{\sigma}} \cos \left(\frac{1}{2} \theta \tau\right),
$$

which the former is famous for interference attenuation and the latter is well known for interference concentration. The other form of kernel function can be expressed by

$$
\begin{aligned}
& \Psi_{\mathrm{NEW}}(\mu, \tau)=\frac{1}{2} \frac{\sqrt{\pi \sigma}}{\tau}\left[e^{-\frac{(\mu+\tau / 2)^{2}}{4 \tau^{2} / \sigma}}+e^{-\frac{(\mu-\tau / 2)^{2}}{4 \tau^{2} / \sigma}}\right], \\
& \varphi_{\mathrm{NEW}}(\theta, \eta)=\frac{1}{2} \frac{\sqrt{\pi \sigma}}{\theta}\left[e^{-(\eta+\theta / 2)^{2}}+\frac{(\eta-\theta / 2)^{2}}{4 \theta^{2} / \sigma}+e^{-\frac{\left(\eta \theta^{2} / \sigma\right.}{4}}\right] .
\end{aligned}
$$

From these alternative kernel forms, we can get the
operties of new TFD [2], [8] and [9]. In table 1 we list some famous TFDs. In table 2 we compare the 
properties of new TFD with other famous TFDs. Although it is impossible to satisfy all the desirable properties of TFD because of trade-off, we already make new distribution meet various requirements as much as we can.

Table 1 Some popular distributions and their kernels

TFD

Page $[10]$

Kernel $\Phi(\theta, \tau)$ $e^{j \theta|\tau| / 2}$

Levin's IPS [7] $\cos \left(\frac{1}{2} \theta \tau\right)$

Kirkwood [11] $e^{j \theta \tau / 2}$

$S \operatorname{h}\left(\mu+\frac{1}{2} \tau\right) h^{*}$.

$\left(\mu-\frac{1}{2} \tau\right) e^{-j \theta \mu} d \mu$

WVD, Wigner [12] 1

Ville [13]

Choi, Williams $e^{-\theta^{2} t^{2} / \sigma}$

ED [6]

$$
\int s\left(t+\frac{1}{2} \tau\right) s^{*}\left(t-\frac{1}{2} \tau\right) e^{-j \omega \tau} d
$$$$
\int S \sqrt{\frac{\sigma}{4 \pi \tau^{2}}} e^{-\frac{(\mu-\tau)^{2}}{4 \tau^{2} / \sigma}}
$$

$S\left(\mu+\frac{1}{2} \tau\right) S^{*}\left(\mu-\frac{1}{2} \tau\right) e^{-j \omega \tau}$

Table 2 Some popular distributions and their properties

\begin{tabular}{|c|c|c|c|c|c|}
\hline & IPS & Spectrogram & WVD & $\mathrm{ED}$ & New TFD \\
\hline Shift-invariant & * & * & * & * & * \\
\hline Reality & * & * & * & * & * \\
\hline Scale-invariant & * & & * & * & * \\
\hline Marginal & * & & * & * & * \\
\hline $\begin{array}{l}\text { Mean Conditional } \\
\text { Frequency/Time }\end{array}$ & $*$ & & * & * & * \\
\hline Range of distribution & * & & * & & \\
\hline $\begin{array}{l}\text { Interference } \\
\text { attenuation }\end{array}$ & & * & & * & * \\
\hline $\begin{array}{l}\text { Interference } \\
\text { concentration }\end{array}$ & * & * & & & * \\
\hline Moyal Formula & & & * & & \\
\hline $\begin{array}{l}\text { W. S. S. } \\
\text { random signal }\end{array}$ & * & & * & * & * \\
\hline
\end{tabular}

Substituting eq.(14) into eq.(1), we get the definition of new TFD,

$$
\mathrm{P}_{\mathrm{NEW}}(t, \omega)=\frac{1}{2 \pi} \delta \int \delta e^{-j \theta t-j \omega \tau+j \theta \mu-\frac{\theta^{2} \tau^{2}}{\sigma}} \cos \frac{\theta \tau}{2} s\left(\mu+\frac{1}{2} \tau\right) \mathrm{s}^{*}
$$$$
\left(\mu-\frac{1}{2} \theta\right) d \mu d \tau d \theta .
$$

Similarly, express in terms of spectrum,

$$
\begin{gathered}
\mathrm{P}_{\mathrm{NEW}}(t, \omega)=\frac{1}{4 \pi^{2}} \iint S e^{-j \theta t-j \omega \tau+j \tau \mu} e^{-\frac{\theta^{2} \tau^{2}}{\sigma}} \cos \frac{\theta \tau}{2} S^{*}\left(\mu+\frac{1}{2} \theta\right) \cdot \\
S\left(\mu-\frac{1}{2} \theta\right) d \mu d \tau d \theta .
\end{gathered}
$$

We can further simplify eq. (17) into more meaning form.

$$
\begin{gathered}
P_{N E W}(t, \omega)=\delta \delta \sqrt{\frac{\sigma}{4 \pi \tau^{2}}} e^{-\frac{(\mu-t)^{2}}{4 \tau^{2} / \sigma_{\frac{1}{2}}\left[s(\mu+\tau) s^{*}(\mu)+S(\mu) s^{*} .\right.}} \\
\left.\left(\mu_{-} \tau\right)\right] e^{-j \omega \tau} \mathrm{d} \mu_{\mathrm{d} \tau}
\end{gathered}
$$

$=\frac{1}{2 \pi} \int S \sqrt{\frac{\sigma}{4 \pi \theta^{2}}} e^{-\frac{(\eta-\omega)^{2}}{4 \theta^{2} / \sigma}} \frac{1}{2}\left[S^{*}(n+\theta) S(\eta)+S^{*}(\eta) S(n-\theta)\right] e^{-j \theta t} d n d \theta$

Recall the definition of Choi Williams ED [6]

$$
\mathrm{P}_{C W}(t, \omega)=\delta \delta \sqrt{\frac{\sigma}{4 \pi \tau^{2}}} \mathrm{e}^{-(\mu-t)^{2} / \sigma} \mathrm{s}\left(\mu+\frac{1}{2} \tau\right) \mathrm{s}^{*}\left(\mu-\frac{1}{2} \tau\right) \mathrm{e}^{-j \omega \tau} \mathrm{d} \mu \mathrm{d} \tau,
$$

and the definition of Levin's IPS [7],

$$
P_{I P S}(t, \omega)=\frac{1}{2} \int\left[s(t+\tau) s^{*}(t)+s(t) s^{*}(t-\tau)\right] e^{-j \omega \tau} d \tau \text {. }
$$

Comparing eq.(19) with (21) and (22), we recognize immediately that, the new TFD is just the mixture of ED and Levin's IPS.

There is a parameter, $\sigma$, in new TED, like ChoiWilliams ED and plays the same role in both cases. In order to obtain sharp auto term resolution, $\sigma$ should be larger. On the other hand, in order to reduce the effects of the cross terms, $\sigma$ should be small. One will note soon that there should be a trade-off between the auto term resolution and the cross term suppression. The optimal chotce of $\sigma$ for a given situation needs more study, but a good choice of $\sigma$ will be found in the range from 0.1 to 10 as will be shown in simulation. When $\sigma$ approaches infinite, $\Phi \mathrm{NEW}(\theta, \tau)$ will approach $\cos (\theta \tau / 2)$, so new TFD approackes Levin's IPS, while Choi-Williams ED approaches WVD. We will conclude that the role new TFD playing respect to Levin's IPS is just the counterpart of Choi-Williams ED respect to WVD.

\section{DISCRETE FORM OF NEW TFD AND COMPUTER SIMULATION}

When one is dealing with sampled signals or digital signals, it is necessary to consider a discrete version of the TFD. The transition from the new TFD of continuous time signals to the new TFD of discrete time signals is not a trivial problem. Several cautions and approximations have to be made for this transition. This section gives the definition of the new TFD for discrete time signals and gives some computer simulations. We derive the following definition of the new TFD for discrete time signals,

$$
\begin{aligned}
P_{N E W}(n, \omega)=\sum_{\tau=-\infty}^{\infty} e^{-j \omega \tau} & \left\{\sum_{\mu=-\infty}^{\infty} \sqrt{\frac{\sigma}{4 \pi \tau^{2}}} e^{-\frac{(\mu-n)^{2}}{4 \tau^{2} / \sigma}} \frac{1}{2}[s(\mu+\tau) .\right. \\
& \left.\left.s^{*}(\mu)+s(\mu) s^{*}(\mu-\tau)\right]\right\} .
\end{aligned}
$$

Compared with the ED [6] for discrete time signals,

$$
P_{C W}(n, \omega)=2 \sum_{\tau=-\infty}^{\infty} e^{-j 2 \omega \tau}\left[\sum_{\mu=-\infty}^{\infty} \sqrt{\frac{\sigma}{4 \pi \tau^{2}}} e^{-\frac{(\mu-n)^{2}}{4 \tau^{2} / \sigma}} s(\mu+\tau) s^{*}(\mu-\tau)\right] \text {, }
$$

the major advantage of new TFD over ED for discrete time signals is

$$
\begin{aligned}
& P_{N E W}(n, \omega)=P_{N E W}(n, \omega+2 \pi), \\
& P_{C W}(n, \omega)=P_{C W}(n, \omega+\pi) .
\end{aligned}
$$

Referring eq.(26), we must double the Nyquist rate to sample the continuous signal to avoid alias. Alternatively, just only for real valued signal, we can use discrete analytic version of signal to avoid alias instead of over-sampling. However, new TFD for discrete time complex valued signal does not need over-sampling still, because its period in frequency is sampling frequency, while ED needs over-sampling. Hence, new TFD is more suitable for complex data application than Choi Williams ED.

For signals consisting of many samples, it is no longer possible to calculate the time-frequency distri- 
bution for the complete signal. That is, for computational purpose it is necessary to apply the weighting windows, $h_{M}(\tau)$ and $h_{M}(\tau)$, for summations in eq. (26) before evaluating the new TFD at each time index $n$. Then, by sliding these windows along the time axis, one can obtain the runing window new TFD, which is defined as follows:

$$
\begin{aligned}
\operatorname{PWNEW}(n, \omega)=\sum_{\tau=-\infty}^{\infty} h_{N}(\tau) e^{-j \omega \tau}\left\{\sum_{\mu=-\infty}^{\infty} h_{M}(\mu) \sqrt{\frac{\sigma}{4 \pi \tau^{2}}} e^{-\frac{(\mu-n)^{2}}{4 \tau^{2} / \sigma}} .\right. \\
\left.\frac{1}{2}\left[s(\mu+\tau) s^{*}(\mu)+S(\mu) s^{*}(\mu-\tau)\right]\right\},
\end{aligned}
$$

where $h_{N}(\tau)$ is a symmetric window, $h_{N}(\tau)=0$ for $|\tau|>N / 2$,

$h_{M}(\mu)$ is a rectangular window, $h_{N}(\mu)=0$ for $|\mu|>M / 2$,

$s(n)$ is discrete analytic version of the signal.

From eq. (27) it is clear that as long as $M$ is large enough, the RWNEW is a smoothed version of the new TFD in the frequency domain, since multiplication in the time domain corresponds to convolution in the frequency domain. That is, the parameter $\mathrm{N}$, the length of the window $h_{N}(\tau)$ and the shape of this window determine the frequency resolution of the RWNEW, while the parameter $M$, the length of the window $h_{M}(\mu)$, determines the range from which the time indexed autocorrelation function is to be estimated. Through experimental observations, it is found that oscillatory fluctuations of the cross terms can be reduced by decreasing the length of the window $h_{N}(\tau)$. On the other hand, it is also noted that the frequency resolution of the auto terms decreases as the length of the window $h_{N}(\tau)$ decreases. That is, the length of the window $h_{N}(\tau)$ determines a trade-off between the high frequency resolution of the auto terms and the smoothed cross terms.

In order to perform a real time analysis, a FFT technique can be utilised for the evaluation of the RWNEW. For this purpose, one can set $\omega=2 \pi \mathrm{k} / \mathrm{N}$. Ther, eq. (27) can be rewritten as follows:

$$
\begin{aligned}
& \operatorname{RWNEW}(n, k)= \sum_{\tau=-\infty}^{\infty} h_{N}(\tau) e^{-j 2 \pi k \tau / N}\left\{\sum_{\mu=-\infty}^{\infty} h_{M}(\mu) \sqrt{\frac{\sigma}{4 \pi \tau^{2}}} e^{-\frac{\mu^{2}}{4 \tau^{2} / \sigma}} .\right. \\
& \frac{1}{2}\left[s(n+\mu+\tau) S^{*}(n+\mu)+s(n+\mu) S^{*}(n+\mu-\tau)\right]
\end{aligned}
$$

Following, we will make some computer simulation. In simulation, we take window function needed in RWED, RWPTD and IPS all to be rectangular window and $M=64$, $\mathrm{N}=64$, excluding PWVD being Hamming window. Three different signals listed below are used in simulations. Simulation 1:

$$
s_{1}(t)=\sin \left[2 x\left(0.4 t+0.7 t^{2}\right)\right]+\sin \left[2 \pi\left(11.6 t-0.7 t^{2}\right)\right] .
$$

Simulation 2:

$$
s_{2}(t)=s_{20}(t)+s_{21}(t),
$$

where

$$
\begin{aligned}
& s_{20}(t)= \begin{cases}\sin \left(0.2 \pi t^{2}\right) & 0 \leq t \leq 32 \\
0 & \text { elsewhere }\end{cases} \\
& s_{21}(t)= \begin{cases}\sin \left[2 \times\left(6 t+0.2 t^{2}\right)\right] & 11.1875 \leq t \leq 27.1875 \\
0 & \text { elsewhere }\end{cases}
\end{aligned}
$$

Simulation 3:

$$
s_{3}(t)=\sin \left[2 \pi\left(0.4 t+0.6 t^{2}\right)\right]+\sin \left[2 \pi\left(0.4 t+0.8 t^{2}\right)\right] .
$$

All signals are sampled to 512-point sequence with $32 \mathrm{~Hz}$ sampling frequency, and the results for various distribution are shown in Fig. 1 to Fig. 10. From these figures, we will clearly observe the role o playing at determining cross term attenuation and auto term sharpness in ED and new TFD. Make the following conclusions:

(1) If $\sigma$ is small, order of $\sigma$ is about to be 1: Both kernels of ED and new TFD will attenuate cross terms effectively, so both distributions look similarly and new TFD's cross term concentration property take a minor benefit. However, the smaller $\sigma$ is, the auto terms of both distribution spread more.

(2) If $\sigma$ is large, order of $\sigma$ is about to be 10: The auto terms of both distribution become sharper than small $\sigma$, while paying cross term attenuation for return. In ED, cross terms will appear obvious gradually, but in new TFD cross terms will be still under control due to cross term concentration property.

(3) If $\sigma$ approach infinite, order of $\sigma$ is about to be 100: ED will approach WVD, while new TFD will approach WVD, while new TFD will approach Levin's IPS.

\section{CONCLUSION}

Time frequency distribution is a powerful tool to analysis time varying signal. However, the cross term effect due to second order form of Cohen's class severely blocks its application. In this paper, we promote a new TFD, which not only meets several useful TFD's requirements but also has good interference immurity. We also induce that the role new TFD playing respect to Levin's IPS is just the counterpart of Choi-Williams ED respect to WVD.

\section{REFERENCE}

[1] L. Cohen, "Generalized phase space distribution functions," J. Math. Phys. vol.7, pp.781-786, 1966.

[2] L. Cohen, "Time frequency distribution - a review," IEEE Proc., vol.77, pp.941-981, 1989.

[3] T.A.C.M. Claaser and W.F.G. Mecklerbrauker, "The Wigner distribution - a tool for time frequency signal analysis; Part 1: continuous time signals," Philips J. Res. vol.35, pp.217-250, 1980.

[4] T.A.C.M. Claaser and W.F.G. Mecklenbrauker, "The Wigner distribution - a tool for time frequency signal analysis; Part 2: discrete time signals," Philips J. Res. vol.35, pp.276-300, 1980.

[5] T.A.C.M. Claasen and W.F.G. Mecklenbrauker, "The Wigner distribution - a tool for time frequency signal analysis; Part 3: relations with other time frequency signal transformations," Philips J. Res. vol.35, pp.372-389, 1980

[6] H.I. Choi and W.J. Williams, "Improved time frequency represertation of multicomponent signals using exponential kernels," IEEE Trans. on A.S.S.P. vol.37, pp.862-871, 1989.

[7] M.J. Levin, "Instantaneous spectra and ambiguity function," IEEE Trans. on Informat. Theory, vol.13, pp.95-97, 1967.

[8] P. Flandrin, "Some features of time frequency representations of multicomponent signal," IEEE Intl. Conf. on A.S.S.P., vol.3, pp.41B.4.1 41B. 4. 4, 1984.

[9] P.J. Loughlin, J.W. Pitton and L.E. Atlas, "New Properties to Alleviate Interference in TimeFrequency Representations," IEEE Intl. Conf. on A.S.S.P., vol.5, pp.3205-3208, 1991.

[10] C.H. Page, "Instantaneous power spectra," J. Appl. Phys., vol.23, pp.103-106, 1952.

[11] J.G. Kirkwood, "Quantum statics of almost classical ensembles, " Phys. Rev., vol.44, pp.31-37, 1933.

[12] E.P. Wigner, "On the quantum correction for thermodynamic equilibrium," Phys. Rev., vol.40, pp.749$759,1932$.

[13] J. Ville, "Theorie et applications de la notion de signal analytigue," Cables at transmission, vol.2A, pp.61-74, 1948. 

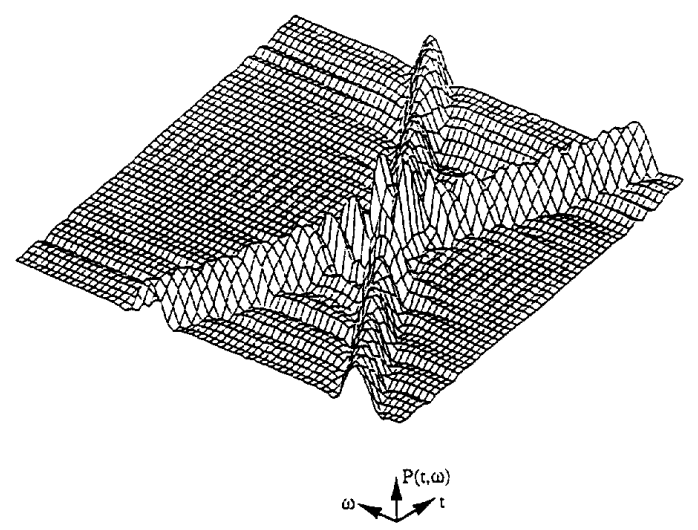

Fig 1 ED of $s_{1}(t)$ with $\sigma=1$
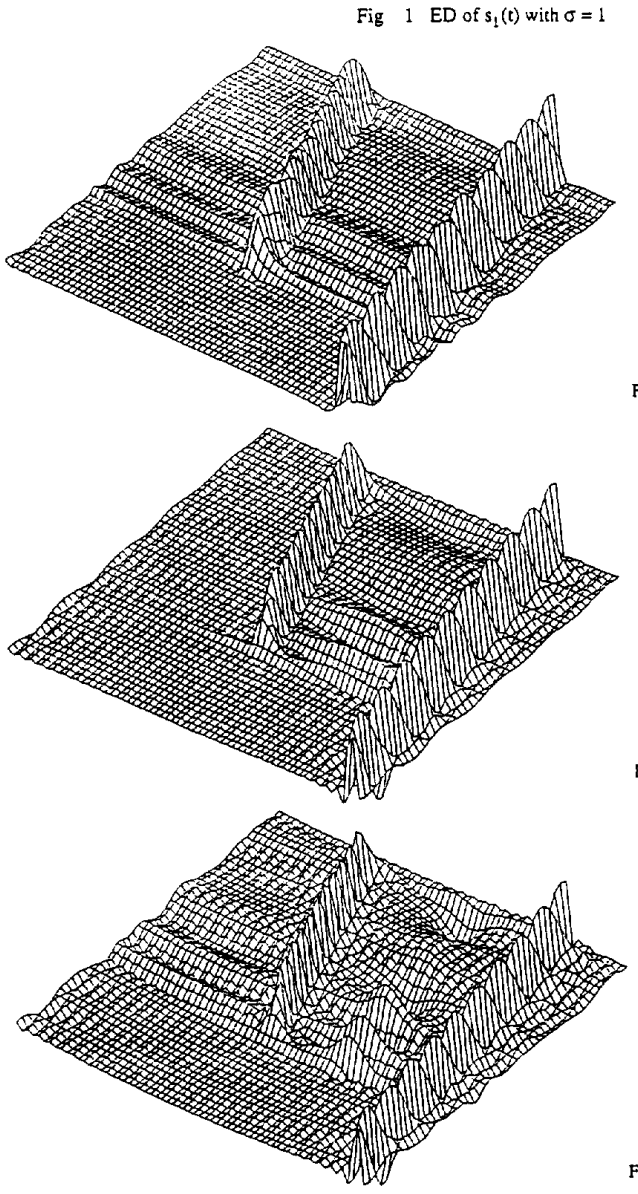

Fig 4 ED of $s_{2}(t)$ with $\sigma=10$

Fig 3 ED of $s_{2}(t)$ with $\sigma=1$

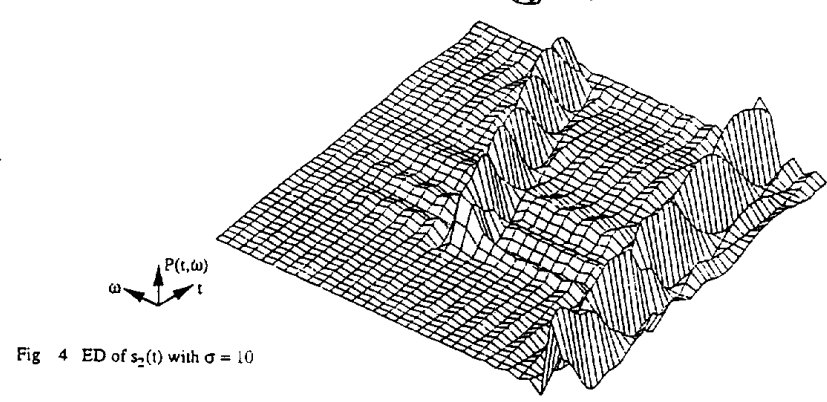

Fig $\int$ ED of $s_{2}(t)$ with $\sigma=10$

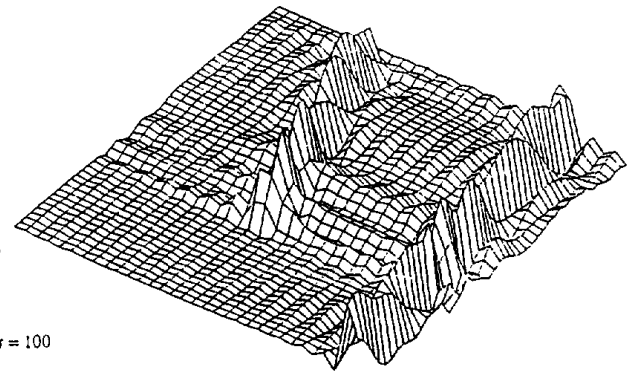

Fig 2 NTD of $s_{1}(t)$ with $\sigma=1$

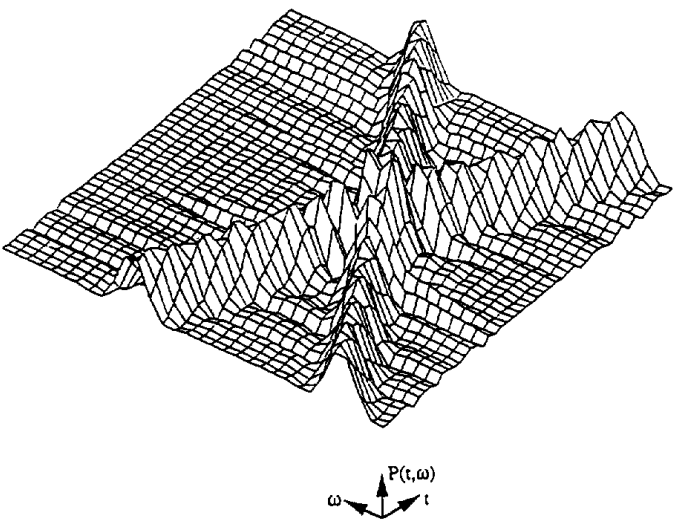

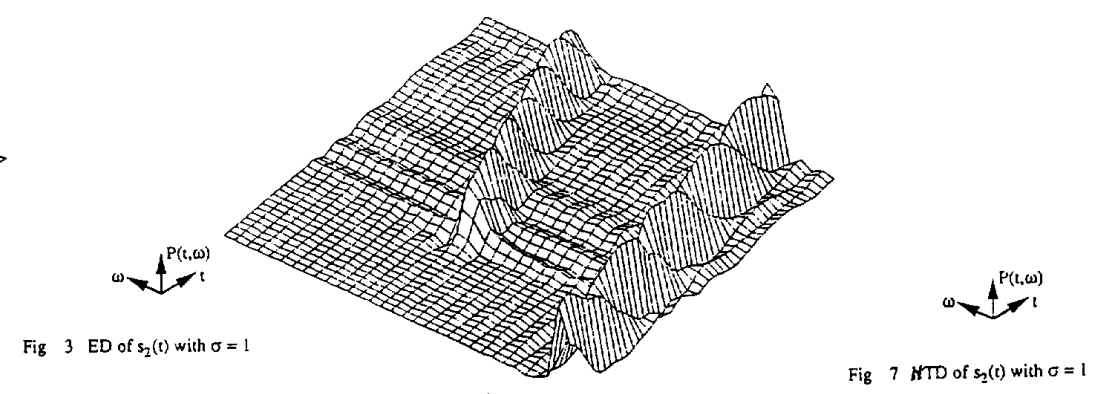

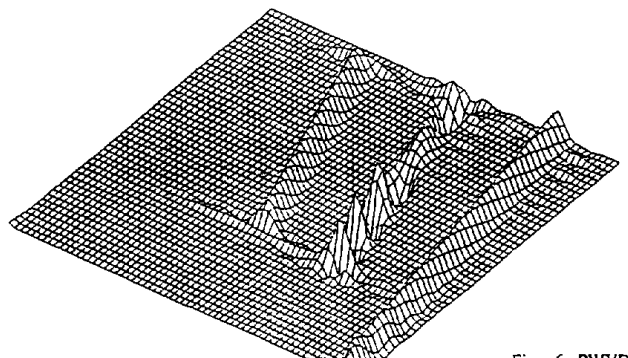

Fig 8 NTD of $s_{2}(t)$ with $\sigma=1$

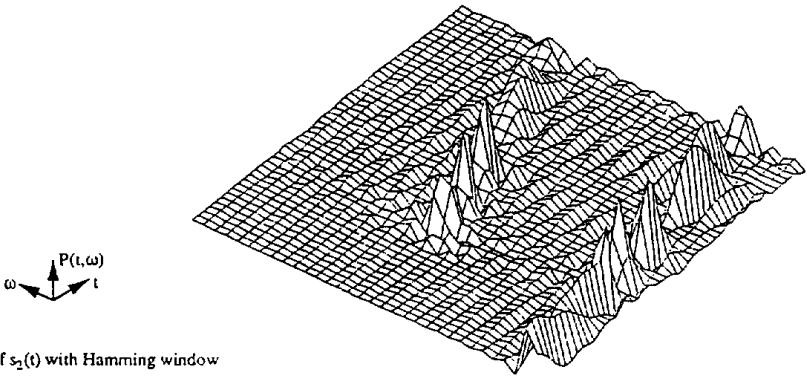

Fig $9 \mathrm{NTD}$ of $s_{2}(t)$ with $\sigma=100$ 